\title{
HEADACHE
}

\section{FAMILY AND SCHOOL FACTORS AND HEADACHE PREVALENCE}

The prevalence of headache in children aged 7-16 years, representing districts of the city of Goteborg with socioeconomic, family, and school variables, is reported from Sahlgrenska Hospital, Goteborg, Sweden. Data obtained by questionnaires from 1297 pupils, representative of the city population, showed $26 \%$ with "headache once a month" and $6 \%$ at daily intervals or several times a week "frequent headache." The prevalence of "headache once a month or more" increased with age and school grade, from $16 \%$ in first grade to $42 \%$ in grade 9 . The prevalence of "frequent" headache, presumably of tension-type, increased from $3 \%$ in second grade to $10 \%$ in third grade. Girls in grades 7-9 were affected more than boys with respect to both types of headache. The risk of frequent headache correlated with class size, increasing with larger classes in lower school, grades 1-3. In intermediate classes, grades 4-6, headache frequency was higher in districts with high unemployment. (Carlsson J. Prevalence of headache in schoolchildren: relation to family and school factors. Acta Paediatr June 1996;85:692-6). (Respond: Dr J Carlsson, Department of Clinical Neuroscience, Neurology Section, Sahlgrenska Hospital, S-41345, Goteborg, Sweden).

COMMENT. The increase in headache frequency in third grade was thought to be related to the larger classes and more targeted schoolwork. The increased prevalence in higher school levels was related to the higher frequency among girls, possibly due to hormonal changes and greater sensitivity to interpersonal conflicts and family stress. Parental separation or divorce and marital problems have been related to recurrent headache in children and adolescents, but these were not risk factors in the present study.

\section{FAMILIAL MIGRAINE WITH VERTIGO AND TREMOR}

A family with dominantly inherited migraine headaches, episodic vertigo, and essential tremor is reported from the UCLA School of Medicine, Los Angeles, CA. Episodes were triggered by stress, exercise, or lack of sleep. Tremor began in adolescence or early adulthood. Treatment with acetazolamide in 5 patients relieved visual auras, headaches, and vertigo, and diminished the tremor. Linkage analysis excluded linkage to markers on chromosome 19p, involved in families with hemiplegic migraine and ataxia syndrome. (Baloh RW et al. Familial migraine with vertigo and essential tremor. Neurology Feb 1996;46:458-460). (Reprints: Dr Robert W Baloh, Department of Neurology, UCLA School of Medicine, Los Angeles, CA 90095).

COMMENT. Several studies have shown a beneficial effect of acetazolamide in essential tremor. In the present report, both tremor and headache were relieved by acetazolamide. Of 15 family members with migraine, 8 also had essential tremor, whereas none of unaffected members had tremor. It seemed likely that the migraine and tremor were genetically connected. Migraine is expressed in various clinical forms and appears to be genetically heterogeneous.

An 11-year-old boy with basilar migraine aura without headache and ictal fast EEG activity is reported from the Pediatric Institute, Ferrara University, Italy (Soriani S et al. Eur I Pediatr Feb 1996;155:126-129). 